\title{
Langmuir
}

pubs.acs.org/Langmuir

(C) 2010 American Chemical Society

\section{Application of Bicellar Systems on Skin: Diffusion and Molecular Organization Effects}

\author{
Gelen Rodríguez, ${ }^{* \dagger}$ Laia Rubio, ${ }^{\dagger}$ Mercedes Cócera,${ }^{\dagger}$ Joan Estelrich, ${ }^{\S}$ Ramon Pons, ${ }^{\dagger}$ \\ Alfonso de la Maza, ${ }^{\dagger}$ and Olga López ${ }^{\dagger}$ \\ ${ }^{\dagger}$ Departament de Tecnologia Química i de Tensioactius, Institut de Quimica Avançada de Catalunya (I.Q.A.C), \\ Consejo Superior de Investigaciones Cientificas (C.S.I.C.), C/Jordi Girona 18-26, 08034 Barcelona, Spain, \\ ${ }^{*}$ BM16, European Synchrotron Radiation Facility, Grenoble, France, and ${ }^{\S}$ Departamento de Fisicoquimica, \\ Facultad de Farmacia, Universidad de Barcelona, Av. Joan XXIII s/n, 08028, Barcelona, Spain
}

Received February 16, 2010. Revised Manuscript Received March 22, 2010

\begin{abstract}
The effect of bicelles formed by dipalmitoylphosphatidylcholine (DPPC)/dihexanoylphosphatidylcholine (DHPC) on stratum corneum (SC) lipids was studied by attenuated total reflectance-Fourier transform infrared (ATR-FTIR) spectroscopy at different temperatures. Analysis of the lipid organization in terms of chain conformational order and lateral packing shows that the use of bicelles hampers the fluidification of SC lipids with temperature and leads to a lateral packing corresponding to a stable hexagonal phase. Grazing incidence small- and wide-angle X-ray scattering (GISAXS and GIWAXS) techniques confirm these results and give evidence of higher lamellar order after treatment with these bicelles. Additionally, the effects of DPPC/DHPC and dimyristoylphosphatidylcholine (DMPC)/DHPC bicelles at different SC depths were compared. The combination of ATR-FTIR spectroscopy and the tape-stripping method was very useful for this purpose.
\end{abstract}

\section{Introduction}

Bicelles are discoidal aggregates formed in water by a flat bilayer of long chain phospholipids, stabilized by a rim of short chain phospholipids. ${ }^{1}$ These structures have the ability to be oriented in magnetic fields, which has permitted their use as membrane models in nuclear magnetic resonance (NMR) studies. ${ }^{2}$ Additionally, bicelles have other properties such as avoiding surfactants, modulable structure, and small enough size for skin penetration. For these reasons, these structures are proposed for new applications such as dermatological, cosmetic, and/or pharmaceutical applications.

The skin barrier function is mainly due to the specific composition and organization of the outermost part of the epidermis, the stratum corneum (SC). This SC is a very thin layer of flat anucleated cells (the corneocytes) surrounding by a lipid matrix organized in bilayers (the intercellular lipids), which are a mixture composed mainly of ceramides, cholesterol, and fatty acids. ${ }^{3-5}$ The molecular organization of the SC lipids has been widely studied, ${ }^{6,7}$ and the effect of bicelles on the skin is currently studied by our group. Recently, we reported that changes in the composition and/or gel-to-liquid crystalline phase transition temperature

*Corresponding author: e-mail gelen.rodriguez@cid.csic.es; Tel 34-93 400 61 00; Fax 34-93 2045904.

(1) Sanders, C. R.; Hare, B. J.; Howard, K. P.; Prestegard, J. H. Prog. NMR Spectrosc. 1994, 26, 421-444.

(2) Whiles, J. A.; Deems, R.; Vold, R. R.; Dennis, E. A. Bioorg. Chem. 2002, 30, 431-442.

(3) López, O.; Cócera, M.; López-Iglesias, C.; Walter, P.; Coderch, L.; Parra, J. L.; de la Maza, A. Langmuir 2002, 18, 7002-7008.

(4) Schaefer, H.; Redelmeier, T. E. Skin Barrier: Principles in Percutaneous Penetration; Karger: Basel, Switzerland, 1996; pp 55-58.

(5) Wertz, P. W.; Downing, D. T. J. Lipid Res. 1983, 24(6), 759-65.

(6) Elias, P. M.; Feingold, K. R. Semin. Dermatol. 1992, 11(2), 176-82.

(7) López, O.; Cócera, M.; Wertz, P. W.; López-Iglesias, C.; de la Maza, A. Biochim. Biophys. Acta 2007, 1768, 521-529.

(8) Barbosa-Barros, L.; Barba, C.; Cócera, M.; Coderch, L.; López-Iglesias, C.; de la Maza, A.; López, O. Int. J. Pharm. 2008, 352, 263-272.
( $\left.T_{\mathrm{m}}\right)$ of phospholipids building bicelles induce specific effects on the skin barrier function. ${ }^{8-10}$

Some of the more useful techniques for these studies are attenuated total reflectance-Fourier transform infrared (ATRFTIR) spectroscopy and X-ray scattering. The vibrational characteristic frequencies of the alkyl chain lipids related to differently ordered phases have been extensively reported by ATR-FTIR. ${ }^{10-12}$ Alkyl chain arrangement of the SC lamellar structure exhibits higher order in the gel phase (orthorhombic, OR, and hexagonal, HEX) than in the liquid-crystalline phase (LIQ). The X-ray scattering technique also provides information about the molecular organization of lipids. ${ }^{13}$ Small-angle X-ray scattering is used to obtain information about the repeat distance of the lipid lamellar phase, and wide-angle X-ray scattering shows the lateral packing of the lipids. In a recent work $^{10}$ we reported useful data on the conformational changes in the SC induced by DMPC/DHPC bicellar systems. These systems caused a phase transition from the gel to the liquid crystalline state in the lipid conformation of SC, a fact probably related to the permeabilizing effect described for the DMPC/DHPC bicelles. ${ }^{8}$ Our previous work shows that the interaction of the bicellar systems with the skin is complex. This complexity is partly based on the versatility of these nanostructures, which have morphologies and structures that are highly sensitive to the thermotropic behavior of the phospholipids. ${ }^{10}$

The present work seeks to evaluate the effect of a new bicellar system, DPPC/DHPC, on the SC lipid organization. This new

(9) Barbosa-Barros, L.; de la Maza, A.; Estelrich, J.; Linares, A. M.; Feliz, M.; Walther, P.; Pons, R.; López, O. Langmuir 2008, 24, 5700-5706.

(10) Rodriguez, G.; Barbosa-Barros, L.; Rubio, L.; Cocera, M.; Diez, A.; Estelrich, J.; Pons, R.; Caelles, J.; De la Maza, A.; Lopez, O. Langmuir 2009, 25(18), 10595-603.

(11) Boncheva, M.; Damien, F.; Normand, V. Biochim. Biophys. Acta 2008, $1778,1344-1355$.

(12) de Jager, M. W.; Gooris, G. S.; Ponec, M.; Bouwstra, J. A. J. Lipid Res. 2005, 46, 2649-2656.

(13) Pereira-Lachataignerais, J.; Pons, R.; Amenitsch, H.; Rappolt, M.; Sartori, B.; López, O. Langmuir 2006, 22(12), 5256-5260. 
system exhibits different thermotropic behavior than that of DMPC due to the melting temperatures of both phospholipids: $23{ }^{\circ} \mathrm{C}$ for DMPC and $41^{\circ} \mathrm{C}$ for DPPC. ${ }^{14}$ That is, at physiological temperature $\left(37^{\circ} \mathrm{C}\right)$, systems containing DMPC exhibits a more fluid state than those containing DPPC. Additionally, the size and morphology of aggregates are also slightly different in both systems. ${ }^{9,15-18}$ These events suggest the different effects of the DMPC/DHPC and DPPC/DHPC systems on the skin lipids. Thus, ATR-FTIR spectroscopy was used here to determine the conformational order of the lipid chains (by analysis of the $\mathrm{CH}_{2}$ stretching mode of the aliphatic chains) and the lateral packing of the SC lipids (by analysis of the $\mathrm{CH}_{2}$ scissoring mode) before and after treatment of the skin with DPPC/DHPC bicelles. X-ray scattering was used to corroborate and to complement results obtained by ATR-FTIR spectroscopy. In addition, a tapestripping method combined with ATR-FTIR spectroscopy was used to evaluate the penetration and effect of this system (DPPC/ DHPC) and the previously studied one (DMPC/DHPC) on the lipid conformational order at different depths of SC. Several authors have reported this methodology as very useful to obtain the variation of the infrared spectra as a function of SC penetration (deeper levels are accessible by removing SC layers by tape stripping). ${ }^{19,20}$

\section{Materials and Methods}

Chemicals. Bicelles were formed by 1,2-dimyristoyl-sn-glycero-3-phosphocholine (DMPC), 1,2-dipalmitoyl-sn-glycero-3phosphocholine (DPPC), and 1,2-dihexanoyl-sn-glycero-3-phosphocholine (DHPC) purchased from Avanti Polar Lipids (Alabaster, AL). Purified water was obtained by an ultrapure water system, Milli-Q plus 185 (Millipore, Bedford, MA). In the washing process on the skin, sodium lauryl ether sulfate (SLES) solution at $0.5 \% \mathrm{w} / \mathrm{v}$ from Sigma-Aldrich Chemie $\mathrm{GmbH}$ (Steinheim, Germany) was used. For studies of penetration, the tape-stripping methodology was performed using adhesive tapestripping (D-squame, CuDem Inc., Dallas, TX).

Skin Preparation. Porcine skin was obtained from the back of experimental animals in the Department of Dermatology, Hospital Clinic of Barcelona (Spain), 2-3 h after the animals were sacrificed. The bristles were removed carefully with an animal clipper, and then the skin was washed with tap water. The excised skin was dermatomed to $500 \pm 50 \mu \mathrm{m}$ thicknesses (Dermatome GA630, Aesculap, Tuttlingen, Germany). Then, full skin containing dermis, epidermis, and SC was used to perform the ATRFTIR experiments. For X-ray scattering experiments the isolation of the epidermis from the dermis was needed. To this end, sections of fresh pig skin were heated with the dermal side in contact with a metal plate for $10 \mathrm{~s}$ at $80^{\circ} \mathrm{C}$, and the epidermis was scraped off in sheets. It has been described that thermal treatments, above $80^{\circ} \mathrm{C}$ for at least $30 \mathrm{~min}$, are able to induce phase transitions in the SC lipid organization, but these transitions are reversible. ${ }^{21}$ Thus, we assume that the method of SC isolation for X-ray scattering did not induce permanent modification in the samples.

(14) Blume, A. Biochemistry 1983, 22, 5436-5442.

(15) Barbosa-Barros, L.; Barba, C.; Rodriguez, G.; Cocera, M.; Coderch, L.; Lopez-Iglesias, C.; de la Maza, A.; Lopez, O. Mol. Pharmaceutics 2009, 6(4), 1237-45.

(16) Dam, L. V.; Karlsson, G.; Edwards, K. Langmuir 2006, 28, 3280-3285.

(17) Sternin, E.; Nizza, D.; Gawrisch, K. Langmuir 2001, 17, 2610-2616.

(18) Triba, M. N.; Devaux, P. F.; Warschawski, D. E. Biophys. J. 2006, 91(4), 1357-67.

(19) Brancaleon, L.; Bamberg, M. P.; Sakamaki, T.; Kollias, N. J. Invest. Dermatol. 2001, 116(3), 380-6.

(20) Coderch, L.; Pera, M. d.; Perez-Cullell, N.; Estelrich, J.; Maza, A. d. 1.; Parra, J. L. Skin Pharmacol. Appl. Skin Physiol. 1999, 12, 235-246.

(21) Ribaud, C.; Garson, J. C.; Doucet, J.; Lévêque, J.-L. Pharm. Res. 1994, 11, 1414-1418.
Preparation and Characterization of the Bicellar Systems. Two bicellar systems, DMPC/DHPC and DPPC/DHPC, were formed at lipid molar ratios of 2 and 3.5, respectively. These two systems were expressly chosen due to the different $T_{\mathrm{m}}(23$ and $41{ }^{\circ} \mathrm{C}$ for DMPC and DPPC, respectively ${ }^{14}$ ) of the long-chain phospholipids and due to the different amounts of the hydrophilic lipid (DHPC) in both cases. These different characteristics could induce different effects on the skin. Samples were prepared by mixing appropriate amounts of either DMPC or DPPC powder and DHPC chloroform solutions to reach a DMPC/DHPC molar ratio 2 and a DPPC/DHPC molar ratio 3.5. After mixing the components, the chloroform was removed with a rotary evaporator, and the systems were hydrated with water to reach $20 \%$ (w/v) of total lipid concentration. Bicellar solutions were prepared by subjecting the sample to several cycles of sonication and freezing until the sample became transparent. ${ }^{22}$ The systems were characterized by dynamic light scattering (DLS) and cryo-transmission electron microscopy (Cryo-TEM) and maintained under refrigeration $\left(\approx 4{ }^{\circ} \mathrm{C}\right)$ until use.

Dynamic Light Scattering. The hydrodynamic diameter (HD) was determined by means of DLS using a Zetasizer Nano ZS90 (Malvern Systems, Southborough, MA), which provided the size distribution curves. The DLS measured the Brownian motion of the particles and correlated it to the particle sizes. The relationship between the size of a particle and its speed due to Brownian motion is defined by the Stokes-Einstein equation:

$$
\mathrm{HD}=k T / 3 \pi \eta D
$$

where HD is the hydrodynamic diameter of a hypothetical hard sphere that diffuses with the same speed as the particle in the experiment, $D$ is the translational diffusion coefficient $\left(\mathrm{m}^{2} / \mathrm{s}\right), k$ is Boltzmann's constant $\left(1.3806503 \times 10^{-23} \mathrm{~J} / \mathrm{K}\right), T$ is the absolute temperature $(\mathrm{K})$, and $\eta$ is the viscosity $(\mathrm{mPa} \cdot \mathrm{s})$.

Because our samples were formed by disks, the simple transformation from hydrodynamic radius $\left(R_{\mathrm{h}}\right)$ to particle radius ( $r$, disk radius) is not possible. The following equation given by Mazer et al. ${ }^{23}$ which relates the $R_{\mathrm{h}}$ with the disk radius was used:

$$
\begin{gathered}
R_{\mathrm{h}}=3 / 2 r\left(\left[1+(t / 2 r)^{2}\right]^{1 / 2}\right. \\
\left.+2 r / t \ln \left[t / 2 r+\left[1+(t / 2 r)^{2}\right]^{1 / 2}\right]-t / 2 r\right)^{-1}
\end{gathered}
$$

For the disk thickness $(t)$, we used a fixed value of $5.4 \mathrm{~nm}$ based on a previous work. ${ }^{9}$ In order to transform the intensity to volume, and considering the small disk radius and small refractive index differences, we can use the Rayleigh-Debye-Gans approximation $^{24}$ and use the form factor for disks in Mazer et al. ${ }^{23}$

The particle sizes were determined by the detection and analysis of scattered light when a $632 \mathrm{~nm} \mathrm{He} / \mathrm{Ne}$ laser beam was incident on the particles. The measurements were performed at several experimental temperatures $\left(32,37\right.$, and $\left.45^{\circ} \mathrm{C}\right)$.

Cryo-Transmission Electron Microscopy (Cryo-TEM). The morphology of the bicellar systems was evaluated by cryoTEM. A thin aqueous film was formed by dipping and withdrawing a bare specimen grid from the suspension. Glowdischarged holey carbon grids were used. After withdrawal from suspension the grid was blotted against filter paper, leaving thin sample films spanning the grid holes. These films were vitrified by plunging the grid into ethane, which was kept at its melting point by liquid nitrogen, ${ }^{25}$ using a Vitrobot (FEI Company, Eindhoven,

(22) Soong, R.; Macdonald, P. M. Langmuir 2009, 25(1), 380-90.

(23) Mazer, N. A.; Benedek, G. B.; Carey, M. C. Biochemistry 1980, 19(4), $601-15$.

(24) Hofer, M. European Workshop on Neutron, X-Ray and Light Scattering as an Investigative Tool for Colloidal and Polymeric Systems In Lindner, P., Zemb, T., Eds.; North Holland Delta Series: Amsterdam, 1991; Vol. Part VI, pp 301-324.

(25) Honeywell-Nguyen, P. L.; Frederik, P. M.; Bomans, P. H.; Junginger, H. E.; Bouwstra, J. A. Pharm. Res. 2002, 19(7), 991-7. 
Netherlands) and keeping the sample before freezing at $100 \%$ humidity. The temperature at which the thin films were kept, where vitrification was initiated, was $37^{\circ} \mathrm{C}$. The vitreous sample films were transferred to a microscope Tecnai F20 (FEI Co., Eindhoven, Netherlands) using a Gatan cryotransfer (Barcelona, Spain). The visualization was taken at $200 \mathrm{kV}$ at a temperature between -170 and $-175^{\circ} \mathrm{C}$ using low-dose imaging conditions. Ten overviews and $\sim 40$ detailed electron micrographs were taken.

Treatment of Skin Tissues with Bicelles. Three disks of whole skin with dimensions of $\sim 2.5 \mathrm{~cm}^{2}$ were used to carry out this experiment. One of the tissues was treated with water (control sample), and the others were treated with one of the two bicellar systems (DMPC/DHPC or DPPC/DHPC bicelles). The treatment was realized placing the skin pieces SC side up, over a thin layer of water (but not submerged) on a Petri dish at $37{ }^{\circ} \mathrm{C}$ controlled by a thermostat. At these specific conditions samples were maintained completely hydrated. The treatment with bicelles or with water consisted of four applications (every $1 \mathrm{~h}$ ) of $13 \mu \mathrm{L}$ of the bicellar system or water on the skin surface. After every application the samples were washed with Milli-Q water and left to dry before the following application. At the end of treatment (four applications), all samples were washed with SLES solution (at $0.5 \% \mathrm{w} / \mathrm{v}$ ) and later with Milli-Q water.

IR Experiments. Infrared spectra of the sample tissue were obtained using a 360-FTIR spectrophotometer Nicolet Avatar (Nicolet Instruments, Inc., Madison, WI) equipped with a $45^{\circ}$ ZnSe thermal horizontal attenuated total reflection (ATR) crystal. All spectra were the average of 256 interferograms, collected within a period of $7 \mathrm{~min}$ at $2 \mathrm{~cm}^{-1}$ of resolution over the 4000$700 \mathrm{~cm}^{-1}$ region.

To collect the IR spectra, the skin sample was placed SC side down onto the ZnSe ATR crystal. In order to ensure reproducible contact between the sample and the crystal, we applied a weight of $260 \mathrm{~g}$ on the samples. The spectra were collected at different temperatures: $32{ }^{\circ} \mathrm{C}$ (skin temperature), $37{ }^{\circ} \mathrm{C}$ (physiological temperature), and $45^{\circ} \mathrm{C}$ (main transition temperature described for SC lipids ${ }^{26-28}$ ). The temperature was controlled by a temperature controller integrated in the ATR device and by an external thermostatic camera. The samples were placed on the equipment $30 \mathrm{~min}$ before collecting the spectra for temperature equilibration.

In order to analyze the original spectra, a fitting procedure was used wherein a number of individual Gaussian peaks were calculated to fit a complex set of overlapping peaks in the spectrum. A Savitsky-Golay second derivate was used to find peaks using the OMNIC software.

Grazing Incidence X-ray Scattering Measurements. All X-ray scattering measurements were carried out using a S3MICRO (Hecus X-ray systems GMBH Graz, Austria) coupled to a GENIX-Fox 3D X-ray source (Xenocs, Grenoble), which provided a detector-focused X-ray beam with a $\lambda=0.1542 \mathrm{~nm} \mathrm{Cu}$ $\mathrm{K} \alpha$ line with more than $97 \%$ purity and less than $0.3 \% \mathrm{~K} \beta$. Two proportional linear detectors, PSD 50 Hecus, were used: one for small angles $\left(0.07 \mathrm{~nm}^{-1}<q<6 \mathrm{~nm}^{-1}\right)$ and one for wide angles $1.3 \mathrm{~nm}^{-1}<q<1.9 \mathrm{~nm}^{-1}$. The GISAXS (grazing incidence smallangle X-ray scattering) and GIWAXS (grazing incidence wideangle X-ray scattering) curves are shown as a function of the scattering vector modulus, $q=4 \pi / \lambda \sin (\theta / 2)$, in which $\theta$ is the scattering angle. The positions of reflections are denoted by their spacing in real space, $d$-spacing, calculated with Bragg's law: $q=$ $2 \pi / d$. The system scattering vector was calibrated by measuring a standard silver behenate sample for the small-angle detector and $o$-parabenzoic acid for the wide-angle detector. Because of the use of a detector-focused small beam $(300 \times 400 \mu \mathrm{m}$ fwhm $)$, the

(26) Chen, X.; Kwak, S.; Lafleur, M.; Bloom, M.; Kitson, N.; Thewalt., J. Langmuir 2007, 23, 5548-5556.

(27) Pensack, R. D.; Michniak, B. B.; Moore, D. J.; Mendelsohn, R. Appl. Spectrosc. 2006, 60, 1399-1404.

(28) Plasencia, I.; Norlén, L.; Bagatolli, L. A. Biophys. J. 2007, 93, 3142-3155.

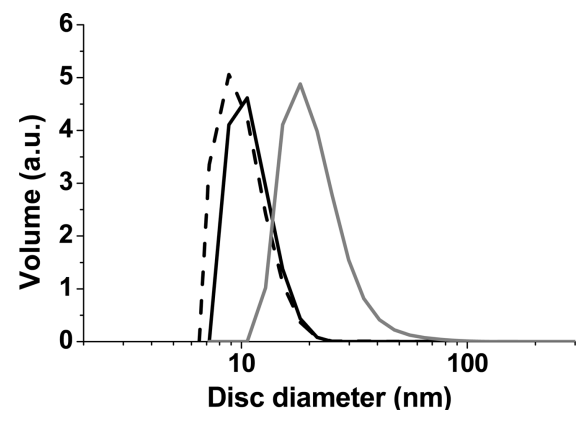

Figure 1. Size distribution curves for the bicellar systems at $32{ }^{\circ} \mathrm{C}$ (long dashed line), $37^{\circ} \mathrm{C}$ (solid line), and $45^{\circ} \mathrm{C}$ (gray line). Size distribution curves were obtained by volume as a function of disk diameter.

scattering curves were mainly smeared by the detector width. This mainly produced a widening of the peaks without noticeable effect on the peak position for $q<0.2 \mathrm{~nm}^{-1}$. Moreover, lamellae oriented parallel to the plane of incidence produce anisotropic scattering, which results in lower smeared scattering curves.

Hydrated samples were mounted by deposition on oxidized silicon (111) cut plane wafers. The wafers were oriented in the scanning direction by a stepping motor with a resolution of $0.01^{\circ}$. A homemade accessory allowed for the use of a humid atmosphere. Humid air at $22^{\circ} \mathrm{C}$ was blown in the sample cell at a $99 \%$ relative humidity. The sample-detector distance was fixed at $268 \mathrm{~mm}$, and the exposure time was between 1800 and $3600 \mathrm{~s}$. The sample was aligned between $0.5^{\circ}$ and $0.25^{\circ}$ of the incident angle.

Tape Stripping. This procedure consists of the removal of SC layers through the sequential application and removal of adhesive tapes. The tape stripping methodology allowed us to know the effect of DMPC/DHPC and DPPC/DHPC bicelles at different depths of SC. The stripping technique was started $2 \mathrm{~h}$ after finishing the treatment of the SC with bicellar systems. Each strip was applied onto the skin with a constant pressure $\left(86 \mathrm{~g} / \mathrm{cm}^{2}\right)$ for $5 \mathrm{~s}$. For each repeated strip, the tearing-off direction was varied by $90^{\circ}$ to obtain a homogeneous removal of the SC cell layers. ATR-FTIR spectroscopy measurements were carried out after $0,4,8,12,16,20$, and 24 strips at $32^{\circ} \mathrm{C}$.

\section{Results}

Bicellar System Characterization. DLS and cryo-TEM techniques were used to characterize the bicellar systems. Figure 1 shows the size distribution curves of DPPC/DHPC systems at 32, 37 , and $45^{\circ} \mathrm{C}$ obtained by volume as a function of disk diameter as described in the Materials and Methods section. Only one peak is observed for each temperature; at $32{ }^{\circ} \mathrm{C}$ (long dashed line), the peak is centered on a population of small particles with a disk diameter of around $8.8 \mathrm{~nm}$. At $37^{\circ} \mathrm{C}$ (solid line), the disk diameter is around $10.6 \mathrm{~nm}$. When the temperature was increased to $45^{\circ} \mathrm{C}$ (gray line), the size of the particles increased slightly and the curve shows a peak at about $18.2 \mathrm{~nm}$. This increased size may be because the temperature is over the $T_{\mathrm{m}}$ of DPPC, which is $41^{\circ} \mathrm{C}$. Thus, the alkyl chains in the bicelles at $45^{\circ} \mathrm{C}$ are in the liquid state, which may induce some morphological changes in the structures. ${ }^{29}$ According to the intensity weighing results, a population centered around $12 \mathrm{~nm}$ and a much larger size population centered around $500 \mathrm{~nm}$ could be seen (data not shown). The cryo-TEM technique allowed us to characterize the morphology of the DPPC/DHPC bicellar system. A number of images were analyzed, and no large aggregates were visualized. Thus, the apparent population at large sizes detected by DLS is probably

29) Leonenko, Z. V.; Finot, E.; Ma, H.; Dahms, T. E. S.; Cramb, D. T. Biophys. J. 2004, 86, 3783-3793. 


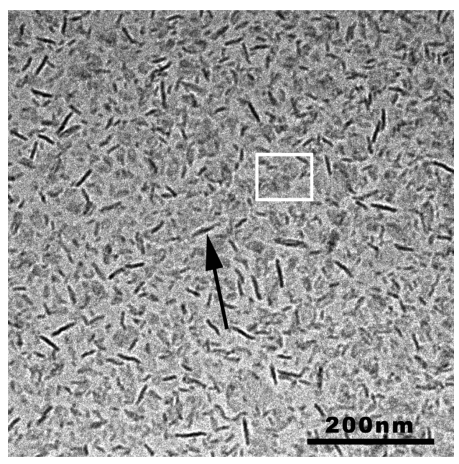

Figure 2. Cryo-TEM micrograph of DPPC/DHPC bicelles at $37^{\circ} \mathrm{C}$. Discoidal structures (bicelles) are shown in all projections, face-on (white square) and edge-on (black arrow).

due to the existence of strong interparticle interactions in the sample. Figure 2 shows one of the cryo-TEM images of DPPC/ DHPC bicelles at $37^{\circ} \mathrm{C}$, in which small discoidal structures with sizes in the range $15-40 \mathrm{~nm}$ were visualized. In addition, bicelles are shown in all projections, face-on (white square) and edge-on (black arrow), although the most evident is the edge-on projection. In order to check the agreement between the results obtained by cryo-TEM and DLS, it is possible to combine the diameters obtained from microscopy $(15-40 \mathrm{~nm})$ with the bilayer thickness $(5.4 \mathrm{~nm})$ obtained by $\mathrm{SAXS}^{9}$ to calculate the hydrodynamic diameter of the particles. Thus, we can approximate the form to an oblate ellipsoid with a major axis between 15 and $40 \mathrm{~nm}$ and a minor axis of $5.4 \mathrm{~nm}$. According to the literature, ${ }^{30} \mathrm{HD} \sim 0.95 \mathrm{DS}$, where DS is the diameter of the sphere with equivalent volume to the disk. With these dimensions, this calculation results in an $\mathrm{HD}$ range between 11 and $22 \mathrm{~nm}$, in agreement with the light scattering results.

Thus, both techniques are complementary in the sense that DLS offers better statistics, whereas cryo-TEM allows for the examination of the morphology of the structures.

DMPC/DHPC bicelles, at a molar ratio of 2 , were also characterized using the same techniques, showing discoidal structures with sizes between 10 and $30 \mathrm{~nm}$ and larger aggregates around $150-250 \mathrm{~nm}$ in the range of $32-45^{\circ} \mathrm{C}$ (data not shown). These results agree fairly well with those reported in previous works. ${ }^{10,31}$ The interaction of DMPC/DHPC structures with the skin lipids was widely described in a previous work. ${ }^{10}$ The present work focused on elucidating the effect of DPPC/DHPC bicelles on SC lipids.

Effect of DPPC/DHPC Bicelles on the SC Lipids. ATR-FTIR. Attenuated total reflectance-Fourier transform infrared spectroscopy (ATR-FTIR) is a technique in which the sample is placed directly onto an infrared crystal. The penetration depth of IR in the skin is about $1 \mu \mathrm{m} .{ }^{20}$ For this reason, this technique allows for the investigation of the SC without isolation from the other skin layers.

The effect of DPPC/DHPC bicelles was evaluated by comparison of the results with those for an untreated sample. Both samples were analyzed at three different temperatures $\left(32,37\right.$, and $\left.45^{\circ} \mathrm{C}\right)$. Spectra analyses focused on bands associated with the alkyl chain of SC lipids vibrations: $\mathrm{CH}_{2}$ stretching (around 2900 and 2850 $\mathrm{cm}^{-1}$ ) and $\mathrm{CH}_{2}$ scissoring (between 1480 and $1460 \mathrm{~cm}^{-1}$ ). ${ }^{11}$

$\mathrm{CH}_{2}$ Stretching Region. This region is particularly important because it provides information about the chain conformational

(30) Schmitz, K. S. In An Introduction to Dynamic Light Scattering by Macromolecules; Press, A., Ed.; Academic Press: San Diego, CA, 1990; p 50.

(31) Marcotte, I.; Auger, M. Concepts Magn. Reson. 2005, 24, 17-37.
Table 1. $\mathrm{CH}_{2}$ Symmetric Stretching $\left(\mathrm{cm}^{-1}\right)^{a}$

\begin{tabular}{ccc}
\hline temperature $\left({ }^{\circ} \mathrm{C}\right)$ & control & treated \\
\hline 32 & 2850.3 & 2850.1 \\
37 & 2851.8 & 2850.4 \\
45 & 2852.5 & 2851.7
\end{tabular}

${ }^{a}$ ATR-FTIR values for the $\mathrm{CH}_{2}$ stretching modes at different temperatures for skin samples before and after treatment with DPPC/ DHPC bicelles.

order of the SC lipids. ${ }^{32,33}$ There are two vibrations associated with the $\mathrm{CH}_{2}$ stretching region: $\mathrm{CH}_{2}$ symmetric and asymmetric vibrations (around 2850 and $2920 \mathrm{~cm}^{-1}$, respectively). This study focused on the $\mathrm{CH}_{2}$ symmetric stretching vibration because it is more sensitive than the asymmetric vibration to packing changes. ${ }^{34}$ Table 1 shows the symmetric vibration for skin samples before and after treatment at different temperatures. Concerning the control sample, the increase of the temperature induces a shift of the $\mathrm{CH}_{2}$ symmetric band to higher values, indicating an order-disorder transition from the HEX phase to the LIQ phase (from 2850.3 to $2852.5 \mathrm{~cm}^{-1}$ ); see Table 1. When skin is treated with DPPC/DHPC bicelles, this shift is slightly smaller. At 32 and $37^{\circ} \mathrm{C}$ the position of the bands remains unaltered, corresponding to a HEX phase. ${ }^{10,11}$ When the temperature was increased $\left(45^{\circ} \mathrm{C}\right)$, a little increase of the value of stretching to $2851.7 \mathrm{~cm}^{-1}$ was detected, corresponding to an intermediate phase between HEX and LIQ. This intermediate state was observed in the control sample already at $37^{\circ} \mathrm{C}$ (Table 1).

$\mathrm{CH}_{2}$ Scissoring Region. The region corresponding to $\mathrm{CH}_{2}$ scissoring vibration, between 1480 and $1460 \mathrm{~cm}^{-1}$, provides information about the lateral packing of lipid alkyl chains. Parts A and $\mathrm{B}$ of Figure 3 show the $\mathrm{CH}_{2}$ scissoring mode from the original spectra of the control sample and the sample treated with DPPC/ DHPC bicelles, respectively. In these panels, from bottom to top, spectra at 32,37 , and $45^{\circ} \mathrm{C}$ are shown. In the control sample at $32{ }^{\circ} \mathrm{C}$ (Figure 3A) two peaks at 1464.7 and $1472.4 \mathrm{~cm}^{-1}$ characteristic of the OR phase are clearly observed. ${ }^{10,11}$ When the temperature reached physiological value $\left(37^{\circ} \mathrm{C}\right)$, a band around $1468 \mathrm{~cm}^{-1}$ could also be observed. This fact is corroborated with curve fitting procedures of this spectrum, which produced three peaks: the characteristic split of the OR phase at 1464.8 and $1472.1 \mathrm{~cm}^{-1}$ and another band at $1467.7 \mathrm{~cm}^{-1}$ corresponding to the HEX phase. ${ }^{10,11}$ At $45{ }^{\circ} \mathrm{C}$, one of the characteristic peaks of the split is lost, and two vibrations are detected at 1472 and $1467.8 \mathrm{~cm}^{-1}$; the latter is the typical HEX band. When DPPC/DHPC bicelles are applied (Figure 3B), only one peak around $1468 \mathrm{~cm}^{-1}$ is observed for all temperatures, which corresponds to a HEX phase of the lipids.

Grazing Incidence X-ray Scattering. Grazing incidence small-angle X-ray scattering (GISAXS) gives information about the larger structural units in the sample, namely the repeat distances ( $d$-spacing) of a lamellar phase, ${ }^{13}$ which in SC studies is related to the lamellar lipid structure. Parts A and B of Figure 4 show GISAXS curves of the control sample and those of the sample treated with DPPC/DHPC, respectively. The control sample (Figure 4A) shows a broad band that could be the overlap of two reflections, with scattering angle values $(q)$ at 0.10 and $0.14 \AA^{-1}$, which are related to repeat distances around 62 and $46 \AA$, respectively. In addition, one reflection of lower intensity around $35 \AA\left(q=0.18 \AA^{-1}\right)$ is observed. In the GISAXS pattern of the DPPC/DHPC treated sample (Figure 4B) four sharp peaks

(32) Gooris, G. S.; Bouwstra, J. A. Biophys. J. 2007, 92, 2785-2795.

(33) Prasch, T.; Knübel, G.; Schmidt-Fonk, K.; Ortanderl, S.; Nieveler, S.; Förster, T. Int. J. Cosmet. Sci. 2000, 22, 371-383.

(34) Mendelsohn, R.; Flach, C. R.; Moore, D. J. Biochim. Biophys. Acta 2006 $1758,923-933$. 

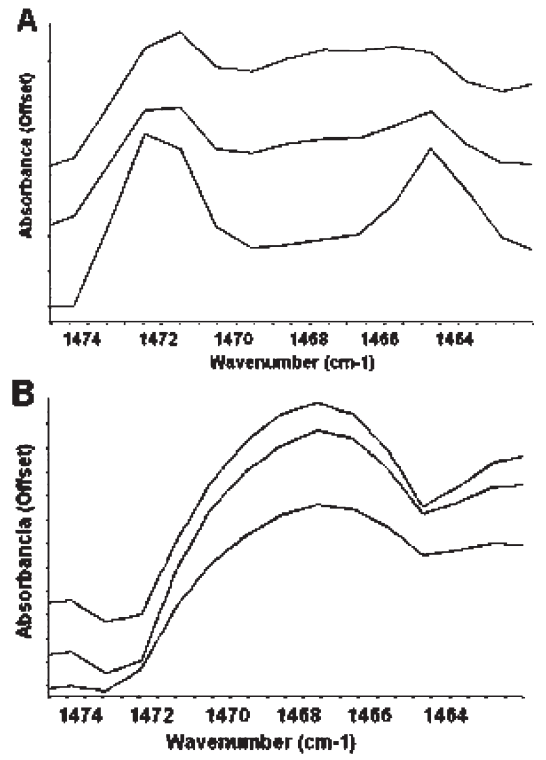

Figure 3. $\mathrm{CH}_{2}$ scissoring mode from original spectra of control sample (A) and sample treated with DPPC/DHPC bicelles (B). The effect of the temperature over each sample, from bottom to top spectra at 32,37 , and $45^{\circ} \mathrm{C}$, is plotted in the panels.

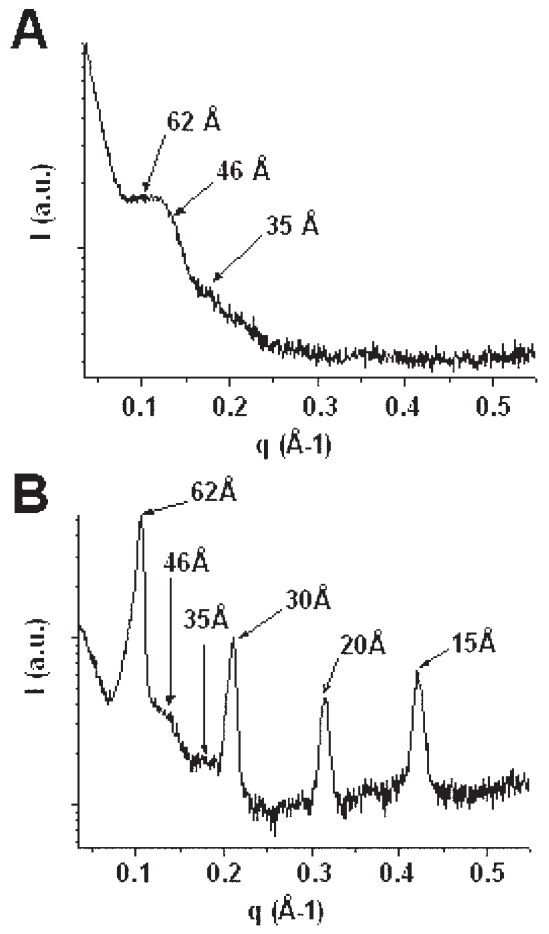

Figure 4. GISAXS curves of control sample (A) and sample treated with DPPC/DHPC bicelles (B).

(at $q=0.10,0.21,0.31$, and $0.42 \AA^{-1}$ ) that correspond to the distances at $62,30,20$, and $15 \AA$ are clearly observed. These reflections could correspond to the first, second, third, and fourth order of a lamellar phase with a periodicity of about $60 \AA$. This diffraction pattern, showing strong peaks, is compatible with a highly ordered lamellar structure in contrast with the weaker scattering bands detected in the control sample. This fact denotes the powerful interaction of bicelles with skin. Furthermore, one shoulder around $46 \AA$ and a small band about $35 \AA$ are observed, which are also found in the control sample. According to Jager et al., ${ }^{12}$ lipids of native SC are organized in two lamellar phases: one
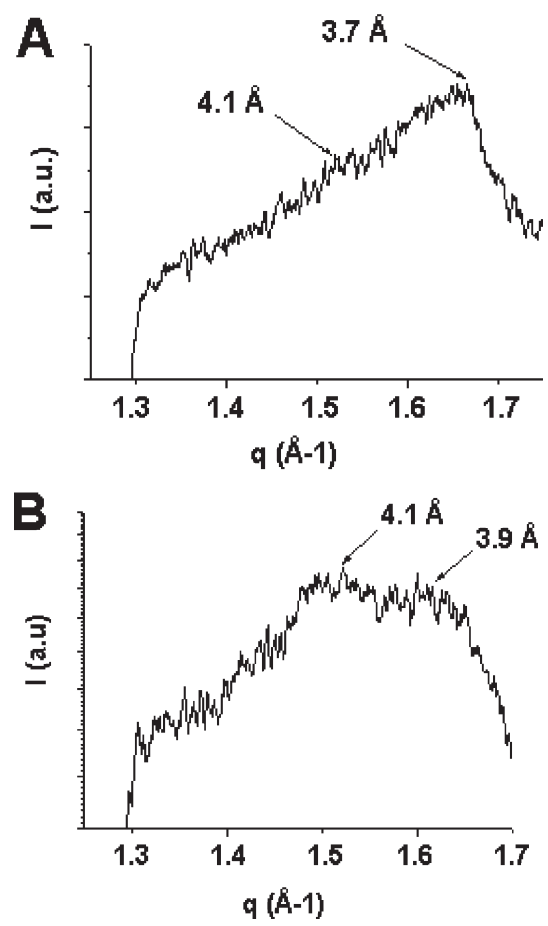

Figure 5. GIWAXS curves of control sample (A) and sample treated with DPPC/DHPC bicelles (B).

with a repeat distance around $135 \AA$, which corresponds with the large lamellar phase, and the other one around $60 \AA$, which corresponds with the short lamellar phase. Our results agree with these data. The reflection we found around $62 \mathrm{~A}$ corresponds to the short lamellar phase in both samples, although for the treated sample, this reflection is observed as a sharp peak, whereas in the control it appears as a broad band. This effect was possibly caused by the interaction of bicelles with the SC lamellar structure. The reflection detected at $46 \AA$ could be the third order of the large lamellar phase at $d=135 \AA$. The reflection around $35 \AA(q=$ $0.18 \AA^{-1}$ ) could correspond to the crystalline cholesterol ${ }^{35}$ or with the fourth order of the larger lamellar phase at $d=135 \AA$. Both of these two reflections, around 46 and $35 \AA$, almost did not change after treatment with bicelles. Thus, our results indicate that only the short lamellar phase was affected by the treatment with bicelles.

Grazing incidence wide-angle X-ray scattering (GIWAXS) provides information about the scattered intensity at higher angles. Thus, information about the smaller structural units in the sample such as the lateral packing in a lamellar phase was acquired. Figure 5 shows GIWAXS profiles for SC before (A) and after (B) treatment with DPPC/DHPC bicelles. Control sample (Figure 5A) shows a X-ray scattering spectrum compatible with two reflections at $q$ values around 1.5 and $1.7 \AA^{-1}$ which correspond to $d$-spacings of 4.1 and $3.7 \AA$, respectively. After treatment (Figure 5B), these reflections are more clearly distinguished with values about 4.1 and $3.9 \AA$. According to Pilgram et al., ${ }^{36}$ the reflections at distances of 4.1 and $3.7 \AA$ are characteristic of lipids in an OR phase. Thus, our results indicate the presence of an OR phase in the control sample and a slight modification of this phase after treatment.

Penetration of DMPC/DHPC and DPPC/DHPC Bicelles through SC. In order to study the effect of bicelles at different depths of the SC, ATR-FTIR measurements after $0,4,8$,

(35) Caussin, J.; Gooris, G. S.; Janssens, M.; Bouwstra, J. A. Biochim. Biophys. Acta 2008, 1778, 1472-1482.

(36) Pilgram, G. S. K.; Petl, A. M. E.; Koerten, H. K.; Bouwstra, J. A. Pharm. Res. 2000, 17, 796-802. 


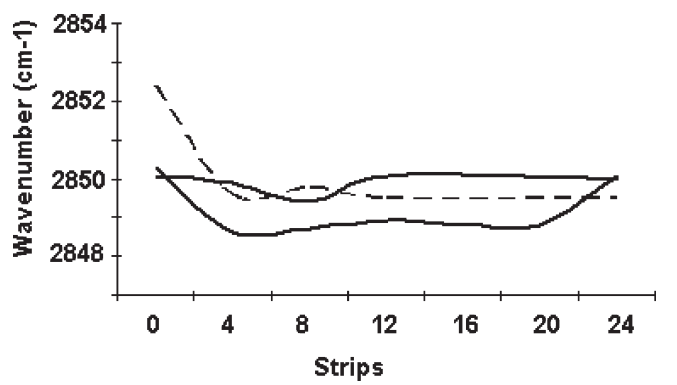

Figure 6. Variation in the $\mathrm{CH}_{2}$ stretching vibration after $0,4,8,12$, 16, 20, and 24 tape strippings for control sample (solid line), sample treated with DPPC/DHPC bicelles (gray line), and sample treated with DMPC/DHPC bicelles (long dashed line).

$12,16,20$, and 24 tape strips were performed. Two regions of the spectrum, $\mathrm{PO}_{2}^{-}$and $\mathrm{CH}_{2}$ stretching vibrations, were analyzed.

The first region (phosphate vibration, $\mathrm{PO}_{2}{ }^{-}$) is characteristic of phospholipids from bicelles because native SC lacks phospholipids and/or any phosphate sources. This vibration was not observed in the sample treated with DPPC/DHPC bicelles regardless of the depth evaluated. However, in the sample treated with DMPC/DHPC bicelles the $\mathrm{PO}_{2}{ }^{-}$vibration was detected only at the outer layer of the tissue (data not shown).

Variations in the $\mathrm{CH}_{2}$ stretching vibration after strippings are shown in Figure 6. The control sample (solid line) and the sample treated with DPPC/DHPC bicelles (gray line) at 0 strips show a similar $\mathrm{CH}_{2}$ stretching vibration, around $2850 \mathrm{~cm}^{-1}$, indicating the presence of lipids in the HEX phase on the skin surface (mentioned previously in the "ATR-FTIR, $\mathrm{CH}_{2}$ stretching region" section). When the sample is treated with DMPC/DHPC bicelles (long dashed line), the $\mathrm{CH}_{2}$ stretching vibration is higher, $2852.4 \mathrm{~cm}^{-1}$, a characteristic vibration of the LIQ phase. ${ }^{10}$ If deeper layers of SC are analyzed after sequential stripping, from 4 to 20 strippings, the $\mathrm{CH}_{2}$ stretching vibration of the control sample is shifted to lower wavenumber values, indicating the presence of an OR phase, but after 24 strips, the stretching value increases to the same wavenumber detected at 0 strips, corresponding to the HEX phase. When skin was treated with DPPC/DHPC bicelles, the $\mathrm{CH}_{2}$ stretching value remained constant, compatible with a HEX phase for all depths. The $\mathrm{CH}_{2}$ stretching vibration in skin treated with DMPC/DHPC bicelles also remained unaltered for depths from 4 to 24 strips, corresponding in this case with an OR phase. Regardless, the wavenumber in the treated samples was always higher than in the control sample.

\section{Discussion}

Bicellar Structures. The composition of bicelles greatly impacts the morphology of the system and on their effect on the skin barrier function. Each phospholipid has a specific $T_{\mathrm{m}}$ depending on the length of the alkyl chain and saturation degree. ${ }^{37}$ When this $T_{\mathrm{m}}$ is exceeded, the gel to liquid-crystalline phase transition occurs, and bicellar structures experience some morphological changes. ${ }^{38}$ This effect is evidenced by our DLS results; the increase in the size of DPPC/DHPC structures was clearly detected at $45^{\circ} \mathrm{C}$. This fact was also confirmed by studying DMPC/DHPC systems, which were demonstrated to be morphologically heterogeneous from $32{ }^{\circ} \mathrm{C} .{ }^{10}$ The melting points of DPPC and DMPC have been reported as 41 and $23{ }^{\circ} \mathrm{C}$,

(37) Williams, W. P.; Cunningham, B. A.; Wolfe, D. H.; Derbyshire, G. E.; Mant, G. R.; Bras, W. Biochim. Biophys. Acta 1996, 1284(1), 86-96.

(38) Diller, A.; Loudet, C.; Aussenac, F.; Raffard, G.; Fournier, S.; Laguerre, M.; Grelard, A.; Opella, S. J.; Marassi, F. M.; Dufourc, E. J. Biochimie 2009, 91(6), $744-51$. respectively. ${ }^{14}$ Thus, the changes in size and morphology above $T_{\mathrm{m}}$ are expected. In this work, bicelles were applied on SC at a physiological temperature $\left(37^{\circ} \mathrm{C}\right)$. At this temperature, both systems, DPPC/DHPC and DMPC/DHPC, exhibited a different phase that led to differently sized aggregates. As a consequence, the effect on each of these two systems on the skin was also different as discussed in the following sections.

Organization of SC Lipids. ATR-FTIR analyses from $\mathrm{CH}_{2}$ stretching vibration evidence and confirm the transition from gel to liquid-crystalline phase of the SC lipids when the temperature increases, a fact described previously by several authors. ${ }^{10,27}$ After treatment with DPPC/DHPC bicelles, the transition to liquidcrystalline phase observed in the control sample at $45^{\circ} \mathrm{C}$ was not found. Instead, at this temperature an intermediate phase between the gel and liquid-crystalline phase was detected. This intermediate state was observed in the control sample at $37^{\circ} \mathrm{C}$. That is, the treated sample at $45^{\circ} \mathrm{C}$ exhibited similar behavior as the control sample at $37^{\circ} \mathrm{C}$. Thus, application of DPPC/DHPC systems could inhibit the transition from gel to liquid-crystalline phase in the SC lipids, indicating an increase in the temperature of lipid fluidification. This effect is opposite to that reported in our previous work for DMPC/DHPC systems, which exhibited a tendency to increase the fluidity of the SC lipids. ${ }^{10}$ This different effect could be due to the incorporation of DPPC (lipids in the gel phase at the application temperature) or DMPC (lipids in the liquid crystalline phase at the application temperature) from bicelles on SC. Thus, DPPC interacted with SC lipids, broadening the range of temperatures at which phase transition occurred. Similar modifications in the lipid termotropic behavior have been reported in bilayer membranes formed by palmitoyl sphingomyelin (PSM) with the addition of long-chain ceramides (C12-C24:1 ceramides). ${ }^{39}$

Results from GISAXS demonstrate that the samples treated with DPPC/DHPC bicelles have higher lamellar order than the native sample, as evidenced by an increase in the intensity, sharpness, and number of reflections associated with the short lamellar phase. This fact is interesting if we consider the sandwich model reported by Bouwstra et al., ${ }^{40}$ who proposed a composition of the short lamellar phase rich in cholesterol and in the linoleic moieties of ceramides 1 and 4 . Our results indicate that phospholipids from bicelles have a tendency to be incorporated in this environment, suggesting a reinforcement of the short lamellar phase of the SC. In relation to this, a previous work ${ }^{9}$ reported the skin penetration of DPPC/DHPC bicelles between corneocytes. In that work, cryo-SEM micrographs showed new lamellar structure in the SC after treatment with bicelles. Thus, the treatment with DPPC/DHPC bicelles involves the incorporation of lipids in the gel phase organized as bilayers in the SC. These findings confirm the increase in the order of SC lipids observed by the $\mathrm{CH}_{2}$ stretching vibration. GISAXS results, previously published for SC treated with DMPC/DHPC systems, only showed a slight increase in the intensity of reflections after treatment, evidencing again the different effect induced by the two bicellar systems. ${ }^{10}$

The chain lateral packing of the SC lipids was studied using the $\mathrm{CH}_{2}$ scissoring vibration and GIWAXS technique. Between 22 and $32{ }^{\circ} \mathrm{C}$ the control sample showed an organization predominantly OR according to both techniques. The interpretation of results for the treated sample is more complex. After treatment, the sample showed predominantly a HEX organization when the $\mathrm{CH}_{2}$ scissoring vibration was observed. However, the position of the two broad bands (at 4.1 and $3.9 \AA$ ) shown in

(39) Westerlund, B.; Grandell, P. M.; Isaksson, Y. J.; Slotte, J. P. Eur. Biophys. J. 2009.

(40) Bouwstra, J. A.; Dubbelaar, F. E.; Gooris, G. S.; Ponec, M. Acta Derm. Venereol. Suppl. 2000, 208, 23-30. 
the GIWAXS profile did not match the previously reported bands for the HEX phase exactly. In any case, considering data published by Pilgram et al., ${ }^{36}$ who reported that distances at 4.1 and $3.7 \AA$ are typical of the OR phase, and because our second reflection ( $d$-spacings $3.9 \AA$ ) was slightly shifted from the position relative to the OR phase, we can discard a purely OR organization. Zellmer et al. ${ }^{41}$ prepared liposomes from an extract of human SC lipids and obtained distances of 4.1 and $3.8 \AA$ A using WAXS, which were related to an OR phase. These authors claimed that with the increase of temperature the second reflection was shifted toward higher distances, until the two reflections were melting in one only reflection at $4.1 \AA$, which corresponded to a HEX phase. Additionally, data reported by Williams et al. ${ }^{37}$ from aqueous dispersions of saturated homoacyl PC derivates associated distances at 4.2 and $3.9 \AA$ with a quasi-hexagonal phase. Overall, the position of the two bands in the sample treated with DPPC/DHPC bicelles could be related to the beginning of the phase transition from OR to HEX. Our results demonstrate the existence of a gel phase before and after treatment by both techniques. This finding is interesting with regard to results from a recent work, ${ }^{10}$ which demonstrated that the treatment with DMPC/DHPC bicelles caused fluidification of SC lipids. The present work points to an opposite effect of the DPPC/DHPC systems, a stabilization of the gel phases.

Diffusion of Bicellar Systems. The phosphate vibration in ATR-FTIR spectra after strippings should allow us to monitor the diffusion of lipids from bicelles through the SC. However, this vibration is only detected at the outer layer of SC treated with DMPC/DHPC. This means that the majority of DMPC/DHPC bicelles remained in the outermost part of the SC, whereas DPPC/ DHPC bicelles would penetrate to deeper layers of the SC and were widely distributed in these different layers. Therefore, the amount of phosphate from bicelles was insignificant with respect to the great amount of other compounds forming the SC. Thus, the $\mathrm{PO}_{2}{ }^{-}$ vibration could be hidden by the vibrations from those components.

The $\mathrm{CH}_{2}$ stretching vibration after different tape stripping showed the lipid organization in the deeper regions of the SC. In the control sample some disorder at the skin surface was detected, and the shift of the $\mathrm{CH}_{2}$ stretching vibration to lower wavenumbers after four strippings indicated that the degree of order of the lipids increased at this depth. Subsequent tape strips did not affect the $\mathrm{CH}_{2}$ peak position, which remained essentially constant up to 20 tape strips. Similar behavior was found by Bommannan et al., ${ }^{42}$ who argued that lipids near the surface represent a mixture of intercellular and sebaceous lipids. While intercellular lipids are expected to form ordered domains, sebaceous lipids contain large amounts of relatively low-melting-point fatty acids. ${ }^{43}$ Because the sebaceous lipids are delivered to the skin surface, their impact on the outer layers by inducing some disorder seems reasonable. Another possible explanation for this disorder at the outer layers could be related to the state, packing, and composition of lipid lamellae from the first SC layers. It is known that these layers, which are almost ready to complete the desquamation process, exhibit molecular disorganization, distorted packing, and anomalous lipid composition. ${ }^{44}$ This situation could cause a loss in the degree of order of the outer layers manifested by the $\mathrm{CH}_{2}$ peak position at higher values than those observed for the inner layers.

(41) Zellmer, S.; Zimmermann, I.; Selle, C.; Sternberg, B.; Pohle, W.; Lasch, J. Chem. Phys. Lipids 1998, 94(1), 97-108.

(42) Bommannan, D.; Potts, R. O.; Guy, R. H. J. Invest. Dermatol. 1990, 95(4), 403-8.

(43) Imokawa, G.; Akasaki, S.; Hattori, M.; Yoshizuka, N. J. Invest. Dermatol. 1986, 87(6), 758-61.

(44) Norlén, L. J. Invest. Dermatol. 2001, 117, 830-836.
It is interesting to note the shift to a higher wavelength of the peak position after 24 tape strips. This effect was also observed by Brancaleon et al. ${ }^{19}$ and Bommannan et al. ${ }^{42}$ and suggests that the aliphatic chains are in different conformations at different depths. Variation in the local water content (which might force lipids to assume a closer packing conformation) or changes in the local lipid composition (which may also change the local conformation of the lipid chains) might be the cause for the changes observed.

Our results imply that the $\mathrm{SC}$ is not uniform across the thicknesses studied. The outer few layers seem less cohesive and more disordered than the deeper ones.

Treated samples showed $\mathrm{CH}_{2}$ peaks at higher wavenumbers than the control sample regardless of the depth, probably due to a mixing effect of lipids from bicelles with SC lipids that modifies the order in lateral packing of the lamellar structure of the tissue without promoting any change of phase. The comparison of the variation of the $\mathrm{CH}_{2}$ stretching wavenumbers in samples treated with both bicellar systems along different SC depths (Figure 6) also agrees with the previous finding: DPPC bicelles penetrate deeper than DMPC bicelles. In samples treated with DMPC/ DHPC the effect of fluidification is only observed in the surface of the SC, in a similar way as other authors described the alkanol that exhibits an enhancer effect in skin permeability. ${ }^{45,46}$ This fact indicates a high interaction of the DMPC/DHPC system with the more superficial layers. In the case of treatment with DPPC/DHPC the wavenumber remains almost unaltered through the different layers studied, and this result demonstrates that DPPC/DHPC bicelles did not show a preferential interaction with superficial layers and were widely distributed at deeper layers of the SC.

Data presented in this work shed light on the interaction mechanisms between bicellar systems and SC lipids and, as a consequence, help us to understand why and how bicellar systems affect skin properties. The penetration enhancer effect described for DMPC/DHPC ${ }^{8,47}$ could agree with the fluidification of the outer layers of the SC described in this work, whereas the reinforcement effect in the SC lipid structures described after treatment with DPPC/DHPC systems ${ }^{9}$ could be consequence of the higher penetration of the lipids forming these nanostructures. The different effects induced by the DMPC/DHPC and DPPC/ DHPC systems could be based on the different alkyl chain lengths of the DMPC and DPPC molecules. The difference in lengths causes different phases of lipids and morphology of aggregates at the physiological temperature, as mentioned above. Additionally, it is of particular interest that these systems were formed using different molar ratios, with the DMPC/DHPC system richer than DHPC. The short chain length of this phospholipid confers some surfactant effects to this molecule, ${ }^{48}$ which could also be involved in the different effects of the DMPC/DHPC system on the skin permeability. Similarly, Novotny et al. ${ }^{49,50}$ have reported on the permeabilizing effect on the skin of short-chained ceramides.

Acknowledgment. This work was supported by funds from CICYT (CTQ 2007-60409) and from Generalitat de Catalunya (GC BIOPOLIM-03086, 2005SR00066).

(45) Cotte, M.; Dumas, P.; Besnard, M.; Tchoreloff, P.; Walter, P. J. Controlled Release 2004, 97, 269-281.

(46) Dias, M.; Naik, A.; Guy, R. H.; Hadgraft, J.; Lane, M. E. Eur. J. Pharm. Biopharm. 2008, 69(3), 1171-5.

(47) Rubio, L.; Alonso, C.; Rodriguez, G.; Barbosa-Barros, L.; Coderch, L.; De la Maza, A.; Parra, J. L.; Lopez, O. Int. J. Pharm. 2010, 386(1-2), 108-113.

(48) Hauser, H. Biochim. Biophys. Acta 2000, 1508, 164-181.

(49) Novotný, J.; Janusová, B.; Novotný, M.; Hrabálek, A.; Vávrová, K. Skin Pharmacol. Physiol. 2009, 22, 22-30.

(50) Novotný, M.; Hrabálek, A.; Janusová, B.; Novotný, J.; Vávrová, K. Bioorg. Med. Chem. Lett. 2009, 19, 344-347. 